\title{
Relationship between the diagnostic components of metabolic syndrome (MS) and cognition by ApoE genotype in the elderly
}

\author{
Kang Soo Lee ${ }^{\mathrm{a}, \mathrm{b}}$, Yangsoo Jang ${ }^{\mathrm{c}, \mathrm{d}}$, Young-Ki Chung ${ }^{\mathrm{e}}$, Ji Hyung Chung ${ }^{\mathrm{c}, \mathrm{d}}$, Byoung Hoon Oh ${ }^{\mathrm{a}, \mathrm{b}}$, \\ Chang Hyung Hong e,f,* \\ a Department of Psychiatry, Yonsei University College of Medicine, Sungsan-ro 262, Seodaemun-gu, Shinchon-dong, Seoul 120-752, South Korea

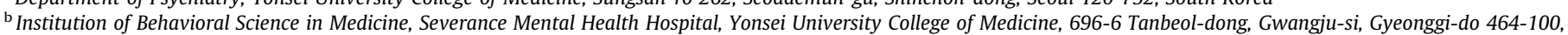 \\ South Korea \\ ${ }^{c}$ Yonsei Research Institute of Aging Science, Yonsei University, Sungsan-ro 262, Seodaemun-gu, Shinchon-dong, Seoul 120-752, South Korea \\ ${ }^{\mathrm{d}}$ Cardiovascular Research Institute, Yonsei University College of Medicine, Sungsan-ro 262, Seodaemun-gu, Shinchon-dong, Seoul 120-752, South Korea \\ e Department of Psychiatry, Ajou University School of Medicine, San 5, Woncheon-dong, Yeongtong-gu, Suwon-si, Gyeonggi-do 443-749, South Korea \\ ${ }^{\mathrm{f}}$ Memory Impairment Center, Ajou University Hospital, San 5, Woncheon-dong, Yeongtong-gu, Suwon-si, Gyeonggi-do 443-749, South Korea
}

A R T I C L E I N F O

\section{Article history:}

Received 1 September 2008

Received in revised form 10 January 2009

Accepted 19 January 2009

Available online 24 February 2009

\section{Keywords:}

Metabolic syndrome

Cognitive impairment

Mini-mental state examination

ApoE genotype in the elderly

\begin{abstract}
A B S T R A C T
The purpose of this study was to find out the effect of the ApoE genotype on the relationship between metabolic syndrome (MS) and its diagnostic components and cognitive impairment in the elderly. A total of 2944 subjects aged over 60 years were analyzed from the data of Gwangju Dementia and Mild Cognitive Impairment Study. We examined demographic characteristics, current and past illness history, drug history, Korean version-mini-mental state examination (K-MMSE). We also examined ApoE genotype and analyzed associated factors with MS. The MS was present in $53.8 \%$ of the subjects (36.8\% of men and $61.1 \%$ of women). On multiple logistic regression analysis, MS was not associated with the cognitive impairment (K-MMSE score $<18$ ) adjusted for age, sex, and educational level. The interactive effect between systolic and diastolic blood pressure (SBP, and DBP, respectively) and ApoE on cognition was not significant (all $p>0.3$ ), but the interactive effect between triglyceride (TG), high-densitylipoprotein-cholesterol (HDLc) and ApoE on cognition was significant after adjustment for age, sex, and education $(B=-0.285$, Wald $=4.194, p=0.041 ; B=0.372$, Wald $=4.134, p=0.042)$. These results suggest that blood TG and HDLc may affect cognitive function in the elderly in the presence of ApoE $\varepsilon 4$ allele. (c) 2009 Elsevier Ireland Ltd. All rights reserved.
\end{abstract}

\section{Introduction}

In elderly populations, the effect of MS on cognitive function may vary depending on the ApoE genotype. Until recently, certain studies reported that cognitive impairment in the elderly was more severe for those with MS (Kalmijn et al., 2000; Yaffe et al., 2004). Individual diagnostic components for MS including hypertension (Haan et al., 1999; Hebert et al., 2004; Qiu et al., 2005; Waldstein et al., 2005), diabetes (Elias et al., 1997; Stewart and Liolitsa, 1999), dyslipidemia (Isbir et al., 2001; Yaffe et al., 2002), and obesity (Jeong et al., 2005) have been shown to increase the risk of dementia or cognitive impairment. Furthermore, the respective effects of each component on cognitive function reportedly differ according to the ApoE genotype (Haan et al., 1999; Stewart and Liolitsa, 1999; Isbir et al., 2001; Yaffe et al.,

\footnotetext{
* Corresponding author at: Department of Psychiatry, Ajou University School of Medicine, San 5, Woncheon-dong, Yeongtong-gu, Suwon-si, Gyeonggi-do 443-749, South Korea. Tel.: +82 31219 5180; fax: +82 312195179 .

E-mail address: antiaging@ajou.ac.kr (C.H. Hong).
}

2002). However, our review of the academic literature has revealed few studies discussing the influence of the ApoE genotype on the relationship between the MS, its component and cognition simultaneously in a community resident elderly population on a large scale. As such, we initiated this study to investigate whether MS and its components are associated with cognitive impairment and modified by the ApoE genotype. Our hypothesis was that the presence of MS or its diagnostic components would be associated with a greater risk of cognitive impairment and that this association would be modified by the ApoE genotype.

\section{Subjects and methods}

\subsection{Study participants}

This study is a part of a large, longitudinal study of people with men aged 60 years or older in the Korean community. Methods are detailed elsewhere (Lee et al., 2009). Briefly, we excluded 341 subjects who fulfilled the exclusion criteria (any difficulty with regard to activities of daily living (ADL); inability to communicate with the interviewer; undergoing active treatment for cancer in 
the last 5 years; intake of antidepressants, sedatives, or other psychiatric drugs; and suffering from epilepsy or other psychiatric disorders), and 293 subjects who had incomplete data. A total of 2944 subjects were included the analysis; Informed consent was obtained after providing a complete description of the study to the subjects and their relatives. This study has been approved by the Severance Mental Health Hospital Institutional Review Board.

\subsection{Assessment and measurements}

Considering the difficulties faced by the elderly in answering self-report questions, all surveys were conducted through the assistance of investigators. The investigators, who had experience in epidemiologic studies, were trained by senior psychiatrists and neuropsychologists to be familiar with the administration of the questionnaires to the study subjects. Information was gathered on the participant's age, sex, educational level, literacy, marital status, living arrangements (i.e., the number of family members living together), medical history, and history of drug, alcohol and tobacco use. The information was verified from at least one close family member or reliable informants closely acquainted with the subject. Physical measurements were taken with the assistance of two nurses. For waist circumference, the narrowest area between the upper end of the ileac crest and the lower end of the scapula was measured while the subject stood upright. Fasting blood samples were obtained in the morning.

\subsection{Definition of cognitive impairment}

Assessing cognitive function with the K-MMSE (Kang et al., 1997), we defined cognitive impairment by a K-MMSE score lower than 18. A Korean study in the community defined the cut-off point of K-MMSE score for screening of dementia as $17 / 18$ points; the sensitivity and specificity of the findings were $91 \%$ and $86 \%$, respectively (Kim et al., 2003).

\subsection{Definition of $M S$}

In the present study, MS was defined according to the modified NCEP-ATP III standard. Cases satisfying three or more of the five following diagnostic criteria were deemed to constitute MS: (a) abdominal obesity: waist circumference in excess of $90 \mathrm{~cm}$ (male) or $80 \mathrm{~cm}$ (female); (b) hypertension: systolic blood pressure higher than $130 \mathrm{~mm} / \mathrm{Hg}$ or diastolic blood pressure higher than $85 \mathrm{~mm} /$ $\mathrm{Hg}$ or currently using an antihypertensive medication; (c) hyperglycemia: fasting blood glucose level higher than $110 \mathrm{mg} /$ $\mathrm{dl}$ or currently using antidiabetic medication (e.g., insulin or oral agents); (d) hypertriglyceridemia: TG level higher than $150 \mathrm{mg} / \mathrm{dl}$; and (e) hypo-HDLc-emia: HDLc level less than $40 \mathrm{mg} / \mathrm{dl}$ (male) or $50 \mathrm{mg} / \mathrm{dl}$ (female).

\subsection{Statistical analysis}

The data were analyzed as follows. Categorical variables were compared by the $\chi^{2}$-test and continuous variables by the Student $t$ test for MS. After adjusting for age, gender, and education level multiple logistic regression analysis was performed for cognitive impairment (K-MMSE score $<18$ ) with MS by the ApoE genotype. To test the influence of the ApoE genotype on the relationship between MS and diagnostic components of MS and cognition, point biserial correlation between cognitive impairment and the diagnostic components of MS was performed according to the ApoE genotype. To verify the interactive effects between each diagnostic component and the ApoE genotype on cognitive impairment, we performed a multiple logistic regression analysis after adjusting for age, gender and education level. The ApoE genotype was transformed by contrast coding, while each diagnostic component was transformed to a standardized score. Interaction term was included in the multiple logistic regression model by multiplying the ApoE genotype by each diagnostic component as an independent variable. In all statistical analyses, the significant level was $p<0.05$. As a statistical program, the SPSS 12.0 version was used.

\section{Results}

\subsection{Demographic characteristics of subjects}

The baseline demographic characteristics of the subjects according are described in Table 1 . One thousand five hundred

Table 1

Demographic characteristics of the subject population by presence of MS, mean \pm S.D. or $n$ (\%).

\begin{tabular}{|c|c|c|c|c|}
\hline Variables & Total $(n=2944)$ & Without MS $(n=1359)$ & With MS $(n=1585)$ & $p$ \\
\hline \multicolumn{5}{|l|}{ Sex } \\
\hline Males & $883(30.0)$ & $558(63.2)$ & $325(36.8)$ & \multirow[t]{2}{*}{$<0.0001^{\mathrm{b}}$} \\
\hline Females & $2061(70.0)$ & 801 (38.9) & $1260(61.1)$ & \\
\hline Age (years) & $72.1 \pm 6.7$ & $71.9 \pm 6.9$ & $72.3 \pm 6.4$ & \multirow{4}{*}{$\begin{array}{l}0.09^{\mathrm{a}} \\
0.14^{\mathrm{b}}\end{array}$} \\
\hline $60-69$ & $1095(37.2)$ & $530(48.4)$ & $565(51.6)$ & \\
\hline $70-79$ & $1413(48.0)$ & $628(44.5)$ & $785(55.5)$ & \\
\hline $80-$ & $436(14.8)$ & $201(46.1)$ & $235(53.9)$ & \\
\hline Education (years) & $4.8 \pm 4.5$ & $5.5 \pm 4.8$ & $4.2 \pm 4.3$ & $<0.0001^{\mathrm{a}}$ \\
\hline \multicolumn{5}{|l|}{ Literacy } \\
\hline No & $548(18.6)$ & $206(37.6)$ & $342(62.4)$ & \multirow[t]{2}{*}{$<0.0001^{\mathrm{b}}$} \\
\hline Yes & $2396(81.4)$ & $1153(48.1)$ & $1243(51.9)$ & \\
\hline \multicolumn{5}{|l|}{ Marital status } \\
\hline Married/remarried & $1784(60.6)$ & $892(50.0)$ & $892(50.0)$ & \multirow[t]{2}{*}{$<0.0001^{\mathrm{b}}$} \\
\hline Unmarried/widowed/separated & $1160(39.4)$ & $467(40.3)$ & $693(59.7)$ & \\
\hline Number of family members living together & $2.5 \pm 1.8$ & $2.4 \pm 1.8$ & $2.6 \pm 1.8$ & $0.01^{\mathrm{a}}$ \\
\hline \multicolumn{5}{|l|}{ ApoE $(n=1516)$} \\
\hline$\varepsilon 4(-)$ & $1271(83.3)$ & $576(45.3)$ & $695(54.7)$ & \multirow[t]{2}{*}{$0.44^{\mathrm{b}}$} \\
\hline$\varepsilon 4(+)$ & $245(16.2)$ & $104(42.4)$ & $141(57.6)$ & \\
\hline K-MMSE & $22.0 \pm 4.7$ & $22.4 \pm 4.7$ & $21.6 \pm 4.6$ & $<0.0001^{\mathrm{a}}$ \\
\hline
\end{tabular}

\footnotetext{
a Student $t$-test.
}

b $\chi^{2}$-test. 
Table 2

Point biserial correlation between cognitive impairment (K-MMSE $<18$ ) and the diagnostic components of MS.

\begin{tabular}{lcc}
\hline & ApoE $\varepsilon 4(-)$ & ApoE $\varepsilon 4(+)$ \\
\hline Waist circumference & -0.009 & 0.111 \\
SBP & $0.087^{*}$ & $0.190^{* *}$ \\
DBP & 0.061 & $0.164^{* *}$ \\
FBG & -0.008 & -0.071 \\
HDLc & -0.004 & $-0.171^{* *}$ \\
TG & -0.035 & $0.188^{*}$ \\
\hline${ }^{*} p<0.01$. & & \\
${ }^{* *} p<0.05$. & &
\end{tabular}

eighty-five subjects (53.8\%) had MS. According to the presence of MS, there were significant differences in $\operatorname{sex}\left(\chi^{2}=147.2\right.$, d.f. $=1$, $p<0.0001)$, educational level $(t=7.3$, d.f. $=2762, p<0.0001)$, literacy $\left(\chi^{2}=19.9\right.$, d.f. $\left.=1, p<0.0001\right)$, marital status $\left(\chi^{2}=26.8\right.$, d.f. $=1, p<0.0001)$, and the number of family members living together $(t=-2.45$, d.f. $=2941, p=0.01)$.

\subsection{Relationship between MS and cognitive impairment}

According to the multiple logistic regression analysis, MS was not associated with cognitive impairment after adjusting for age, sex, and educational level. Despite the presence of the ApoE $\varepsilon 4$ allele, MS was not associated with cognitive impairment (K-MMSE score $<18$ ) after adjusting for age, sex, and educational level.

\subsection{Relationship between the diagnostic components of MS and cognitive impairment according to the ApoE genotype}

As indicated in Table 2, point biserial correlation showed that the correlation coefficient between SBP, DBP, HDLc, TG, and cognitive impairment was less than 0.10 in the ApoE e4(-) group but more than 0.10 in the ApoE e4(+) group. Accordingly, for these four variables in the latter group, we performed additional analyses that showed that the interactive effect between SBP, DBP and ApoE on cognition was not significant (all $p>0.3$ ); however, the interactive effect between TG, HDLC and ApoE on cognition was significant after adjusting for age, sex, and education
$(B=-0.285$, Wald $=4.194, \quad p=0.041 ; \quad B=0.372$, Wald $=4.134$, $p=0.042$ ). The results are described in Tables 3 and 4 .

\section{Discussion}

Our study showed that among the elderly over 60 years old in a community, there was no significant association between MS and cognitive impairment (K-MMSE score $<18$ ) after adjustment for age, sex, and educational level. And this non-association remained regardless of the presence of ApoE $\varepsilon 4$ allele. However, previous studies reported that there was a significant association between MS and cognitive decline in the elderly (Dik et al., 2007; Komulainen et al., 2007; Van den Berg et al., 2007; Yaffe et al., 2007). It is possible that due to difference of the research design, different results may be obtained. The former studies were longitudinal studies, on the other hand, our study was a crosssectional study and thus it was difficult to compare them directly with our results. The former studies used different assessments of cognitive function such as 3MS and other neuropsychological test. MS was associated with memory impairment, but not with the MMSE score (Komulainen et al., 2007). Another possibility is that there was a significant interaction between the MS and inflammation on cognition. The MS was negatively associated with cognition in subjects with high inflammation whereas an association was absent in subjects with low inflammation (Dik et al., 2007).

In the present study, the higher blood TG or lower blood HDLc was, the more cognitive impairment (K-MMSE score $<18$ ) was after adjusting for age, sex, and educational level, in the presence of ApoE $\varepsilon 4$ allele. In animal model, hypertriglyceridemia, the main dyslipidemia of MS, is in part responsible for the leptin resistance seen in obesity. So TGs are likely a major cause of the cognitive disturbances in diet-induced obesity (Farr et al., 2008). In human, poorer cognitive performances of individuals with hypertriglyceridemia has been reported in centenarians (Atzmon et al., 2002). Our result is consistent with a previous study reporting that higher TG levels were associated with a decline in memory function. The authors insisted that lipid levels moderated the influence of ApoE on episodic memory, such that decline in recognition was noted for ApoE $\varepsilon 4$ allele carriers with higher cholesterol levels (De Frias et al., 2007).

Table 3

Interactive effect on cognition between ApoE e4 and TG after adjusting for age, sex, and education.

\begin{tabular}{|c|c|c|c|c|c|c|c|}
\hline & $B$ & \pm S.E. & Wald & d.f. & $p$ & $\mathrm{OR}=$ & $95 \% \mathrm{CI}$ for OR \\
\hline Intercept & -9.850 & 1.402 & 49.342 & 1 & $<0.0001$ & 0.000 & \\
\hline Sex & 0.447 & 0.262 & 2.921 & 1 & $<0.087$ & 1.564 & $0.936-2.612$ \\
\hline Age & 0.111 & 0.016 & 46.464 & 1 & $<0.000$ & 1.117 & $1.082-1.153$ \\
\hline Education level & -1.030 & 0.111 & 86.116 & 1 & $<0.000$ & 0.357 & $0.287-0.444$ \\
\hline ApoE e4 & -0.305 & 0.151 & 4.067 & 1 & $<0.044$ & 0.737 & $0.548-0.991$ \\
\hline TG & 0.269 & 0.140 & 3.686 & 1 & $<0.055$ & 1.308 & $0.994-1.721$ \\
\hline ApoE e $4 \times$ TG & 0.285 & 0.139 & 4.194 & 1 & $<0.041$ & 1.329 & $1.012-1.746$ \\
\hline
\end{tabular}

Nagelkerke $R^{2}=0.393$.

Table 4

Interactive effect on cognition between ApoE e4 and HDLc after adjusting for age, sex, and education.

\begin{tabular}{|c|c|c|c|c|c|c|c|}
\hline & $B$ & \pm S.E. & Wald & d.f. & $p$ & $\mathrm{OR}=$ & $95 \% \mathrm{CI}$ for $\mathrm{OR}$ \\
\hline Intercept & 9.854 & 1.403 & 49.337 & 1 & $<0.0001$ & 0.000 & \\
\hline Sex & 0.428 & 0.262 & 2.677 & 1 & $<0.102$ & 1.534 & $0.919-2.561$ \\
\hline Age & 0.111 & 0.016 & 46.312 & 1 & $<0.0001$ & 1.117 & $1.082-1.153$ \\
\hline Education level & 1.036 & 0.111 & 87.397 & 1 & $<0.0000$ & 0.355 & $0.286-0.441$ \\
\hline ApoE e4 & -0.358 & 0.166 & 4.632 & 1 & $<0.031$ & 0.699 & $0.505-0.969$ \\
\hline HDLc & -0.416 & 0.183 & 5.170 & 1 & $<0.023$ & 0.660 & $0.461-0.944$ \\
\hline ApoE e $4 \times$ HDLc & -0.372 & 0.183 & 4.134 & 1 & $<0.042$ & 0.690 & $0.482-0.987$ \\
\hline
\end{tabular}

Nagelkerke $R^{2}=0.394$. 
The ApoE genotype that plays an important role for the metabolism and distribution of cholesterol has been shown to be a genetic risk factor for non-familiar sporadic Alzheimer disease (Corder et al., 1993). Until now, the relationship between HDLc in the cerebro-spinal fluid and HDLc in the blood has not been elucidated yet. However, a study reported that lipoprotein which plays a role in transporting cholesterol is present only as the HDLc type in the cerebro-spinal fluid. And in the brain, the ability of astrocytes that support and protect neurons to secrete cholesterol varies according to the ApoE genotype (Dietschy and Turley, 2001). This may be an example of the mechanism explaining the relationship of HDLc and cognitive function. According to this study, it has been reported that in the cases with the ApoE $\varepsilon 4$ allele, the ability of astrocytes to secrete cholesterol was decreased by 2.4 times than the cases with ApoE $\varepsilon 3$ allele and thus it influenced the metabolism of cholesterol in the brain and the distribution, hence, it may induce the vulnerability of the regeneration of cell membrane of neurons and the neural plasticity, which suggests the necessity of studies on this field in future. Furthermore HDLc play a key role in the protection of LDLc from oxidation. Such activity depends on the presence of apolipoproteins and enzymes such as paraoxonase 1, platelet activating factoracetylhydrolase. The impairment of HDLc antioxidative activity in MS is partly related to an enrichment of small HDLc in TGs and their depletion in cholesteryl esters, to the replacement of apoA-I by serum amyloid $\mathrm{A}$, and to glycation and oxidation of apoA-I (Hansel et al., 2006).

Our study had the following strengths. First, it was the first largescale study on the effect of the ApoE genotype on the relationship between cognitive impairment in the elderly and MS and its diagnostic components. Second, by characterizing the association of MS and its diagnostic components with cognitive impairment according to the ApoE genotype, the study may provide some basic information for the strategy to prevent dementia by the ApoE genotype. The risk factors for dementia and cognitive function, such as hypertension, diabetes, hyperlipidemia, and obesity, could be readily assessed by regular examinations and controlled by, interalia, the correction of lifestyles and drug treatments. Therefore, treatment and control of MS and its diagnostic components may play key roles in the overall strategy to prevent dementia.

Our study had the following limitations. First, because our subjects were not recruited randomly, the possibility of a selection bias may preclude the study population from being an accurate representation of the elderly over 60 years old in a community. Hence, the $53.8 \%$ incidence rate of MS in our study should be interpreted as the positive rate of the study population rather than the prevalence rate representing all the elderly in a community. Second, because the K-MMSE is the most base-level screening test to assess cognitive function, its ability to measure cognitive function impairment clinically is limited. Thus, the results of our study, which were based on data from subjects who had completed the K-MMSE, may be limited. However, subsequent data obtained in the future from neuropsychological tests and diagnostic procedures conducted by psychiatrists will continue to be reported. Third, because there is yet to be definitive diagnostic criteria for MS, the standards, including the NCEP-ATP III standard used in the present study, continue to change. Finally, because this was a cross-sectional study, the general applicability of our results may be limited. We anticipate that these limitations will be adjusted for in future prospective cohort studies.

\section{Conflict of interest statement}

None.

\section{Acknowledgement}

This study was supported by a grant from the Korea Health 21 R\&D Project of the Ministry of Health and Welfare, Republic of Korea (A050079).

\section{References}

Atzmon, G., Gabriely, I., Greiner, W., Davidson, D., Schechter, C., Barzilai, N., 2002. Plasma HDL levels highly correlate with cognitive function in exceptional longevity. J. Gerontol. A: Biol. Sci. Med. Sci. 57, 712-715.

Corder, E.H., Saunders, A.M., Strittmatter, W.J., Schmechel, D.E., Gaskell, P.C., Small, G.W., Roses, A.D., Haines, J.L., Pericak-Vance, M.A., 1993. Gene dose of apolipoprotein E type 4 allele and the risk of Alzheimer's disease in late onset families. Science 261, 921-923.

De Frias, C.M., Bunce, D., Wahlin, A., Adolfsson, R., Sleegers, K., Cruts, M., Van Broeckhoven, C., Nilsson, L.G., 2007. Cholesterol and triglycerides moderate the effect of apolipoprotein E on memory functioning in older adults. J. Gerontol. B: Psychol. Sci. Soc. Sci. 62, 112-118.

Dietschy, J.M., Turley, S.D., 2001. Cholesterol metabolism in the brain. Curr. Opin. Lipidol. 12, 105-112.

Dik, M.G., Jonker, C., Comijs, H.C., Deeg, D.J., Kok, A., Yaffe, K., Penninx, B.W., 2007. Contribution of metabolic syndrome components to cognition in older individuals. Diabetes Care 30, 2655-2660.

Elias, P.K., Elias, M.F., D’Agostino, R.B., Cupples, L.A., Wilson, P.W., Silbershatz, H. Wolf, P.A., 1997. NIDDM and blood pressure as risk factors for poor cognitive performance. The Framingham Study. Diabetes Care 20, 1388-1395.

Farr, S.A., Yamada, K.A., Butterfield, D.A., Abdul, H.M., Xu, L., Miller, N.E., Banks, W.A. Morley, J.E., 2008. Obesity and hypertriglyceridemia produce cognitive impairment. Endocrinology 149, 2628-2636.

Haan, M.N., Shemanski, L., Jagust, W.J., Manolio, T.A., Kuller, L., 1999. The role of APOE $\varepsilon 4$ in modulating effects of other risk factors for cognitive decline in elderly persons. J. Am. Med. Assoc. 282, 40-46.

Hansel, B., Kontush, A., Bonnefont-Rousselot, D., Bruckert, E., Chapman, M.J., 2006. Alterations in lipoprotein defense against oxidative stress in metabolic syndrome. Curr. Artheroscler. Rep. 8, 501-509.

Hebert, L.E., Scherr, P.A., Bennett, D.A., Bienias, J.L., Wilson, R.S., Morris, M.C., Evans, D.A., 2004. Blood pressure and late life cognitive function change: a biracial longitudinal population study. Neurology 62, 2021-2024.

Isbir, T., Agaçhan, B., Yilmaz, H., Aydin, M., Kara, I., Eker, E., Eker, D., 2001. Apolipoprotein-E gene polymorphism and lipid profiles in Alzheimer's disease. Am. J. Alzheimers Dis. Other Demen. 16, 77-81.

Jeong, S.K., Nam, H.S., Son, M.H., Son, E.J., Cho, K.H., 2005. Interactive effect of obesity indexes on cognition. Dement. Geriatr. Cogn. Disord. 19, 91-96.

Kalmijn, S., Foley, D., White, L., Burchfiel, C.M., Curb, J.D., Petrovitch, H., Ross, G.W., Havlik, R.J., Launer, L.J., 2000. Metabolic cardiovascular syndrome and risk of dementia in Japanese-American elderly men. The Honolulu-Asia aging study. Arterioscler. Thromb. Vasc. Biol. 20, 2255-2260.

Kang, Y.W., Na, D.L., Han, S.H., 1997. Validity of K-MMSE in dementia patients. J. Korean Neurol. 15, 300-307.

Kim, J.M., Shin, I.S., Yoon, J.S., Lee, H.Y., 2003. Comparison of diagnostic validities between MMSE-K and K-MMSE for screening of dementia. J. Korean Neuropsychiatr. Assoc. 42, 124-130.

Komulainen, P., Lakka, T.A., Kivipelto, M., Hassinen, M., Helkala, E.L., Haapala, I. Nissinen, A., Rauramaa, R., 2007. Metabolic syndrome and cognitive function: a population-based follow-up study in elderly women. Dement. Geriatr. Cogn. Disord. 23, 29-34.

Lee, K.S., Cheong, H.K., Kim, E.A., Kim, K.R., Oh, B.H., Hong, C.H., 2009. Nutritional risk and cognitive impairment in the elderly. Arch. Gerontol. Geriatr. 48, 95-99.

Qiu, C., Winblad, B., Fratiglioni, L., 2005. The age-dependent relation of blood pressure to cognitive function and dementia. Lancet Neurol. 4, 487-499.

Stewart, R., Liolitsa, D., 1999. Type 2 diabetes mellitus, cognitive impairment and dementia. Diabet. Med. 16, 93-112.

Van den Berg, E., Biessels, G.J., de Craen, A.J., Gussekloo, J., Westendorp, R.G., 2007. The metabolic syndrome is associated with decelerated cognitive decline in the oldest old. Neurology 69, 979-985.

Waldstein, S.R., Giggey, P.P., Thayer, J.F., Zonderman, A.B., 2005. Nonlinear relations of blood pressure to cognitive function: the Baltimore longitudinal study of aging. Hypertension 45, 374-379.

Yaffe, K., Barrett-Connor, E., Lin, F., Grady, D., 2002. Serum lipoprotein levels, statin use, and cognitive function in older women. Arch. Neurol. 59, 378-384.

Yaffe, K., Kanaya, A., Lindquist, K., Simonsick, E.M., Harris, T., Shorr, R.I., Tylavsky, F.A., Newman, A.B., 2004. The metabolic syndrome, inflammation, and risk of cognitive decline. J. Am. Med. Assoc. 292, 2237-2242.

Yaffe, K., Haan, M., Blackwell, T., Cherkasova, E., Whitmer, R.A., West, N., 2007. Metabolic syndrome and cognitive decline in elderly Latinos: findings from the Sacramento Area Latino study of aging study. J. Am. Geriatr. Soc. 55, 758762. 